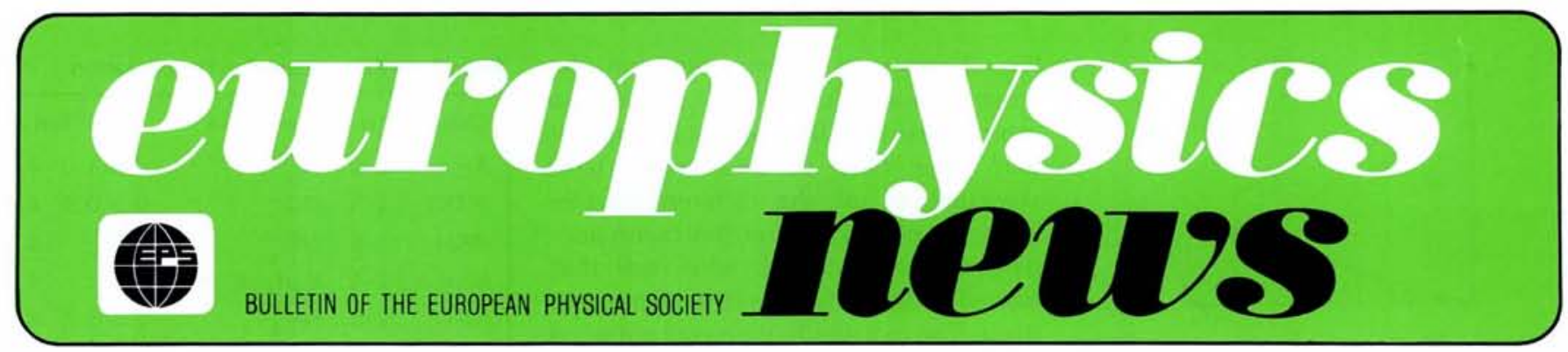

\title{
Properties of Size Selected Neutral Clusters
}

\section{U. Buck, Göttingen}

(Max-Planck-Institut für Strömungsforschung)

Clusters are aggregates of atoms and molecules which are held together by all types of binding force ranging from weak van der Waals and hydrogen bonding to strong metallic, covalent and ionic forces [1]. What make them so attractive for many investigators in different fields, are their unique properties - between those of isolated molecules and bulk condensed matter. It is hoped that their detailed study will improve our understanding of the transition from one to the other with all the implications such as the development of the continuous band structure of the solid out of the discrete spectrum of the molecules, and the change in the absorption of radiation or chemical reactivity as a function of cluster size. In addition, these systems allow us to make a systematic microscopic investigation of important macroscopic phenomena such as nucleation, solvation, and adsorption with many technological applications ranging from catalysis to photography.
Theoretical studies show that clusters of small size do not exhibit the structure of the bulk. Typical van der Waals clusters consist of close-packed icosahedral structures of five-fold symmetry with stable configurations at $n=$ $13,55, \ldots$ and only at very large cluster sizes around $n=1000$ is the transition to the face centred cubic structure of the solid found. For metal clusters, one finds geometrical structures with a preferred stability of $n=8,20, \ldots$ This result can be explained by a theoretical treatment which is quite familiar in physics: the shell model. For metal clusters the delocalized electrons, are embedded in uniform distributions of positive charges, thus combining the rounded potential well of nuclear physics with the jellium of condensed matter physics [2].

Unfortunately, the experimental tools are not so well developed for carrying out the corresponding ideal experiments: the measurement of any desired property of a cluster whose structure is

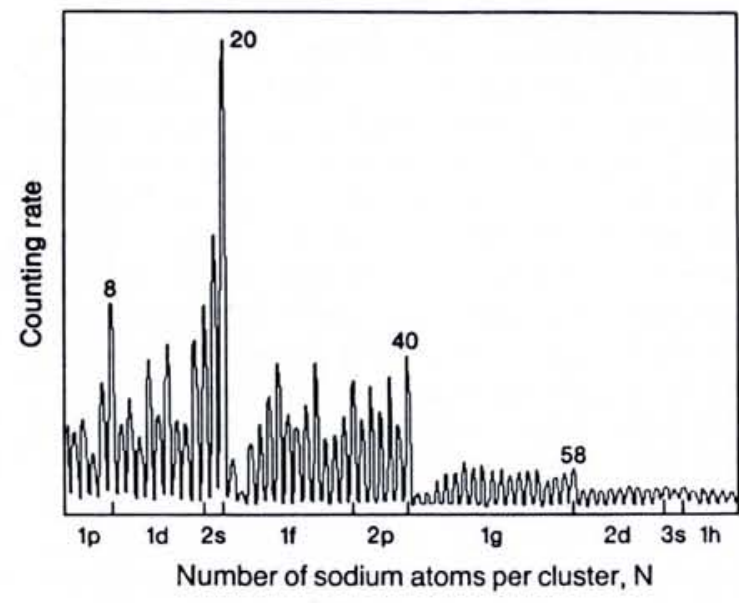

Fig. 1 - Mass abundance spectrum for sodium clusters showing extrema at shell closings according to the spherical shell model for metal clusters (from Ref. 2). well characterized as a function of size. The main reason for this experimental problem is that free clusters have to be generated, and the most commonly used techniques, supersonic isentropic expansion or aggregation in cold gas flows, lead mainly to a spread of cluster sizes. The only way to distinguish experimentally between different cluster species is to use a size-dependent selection process. The straight-forward way to solve this problem is to ionise the clusters and detect the ions in a mass spectrometer. A typical example of such a mass spectrum is shown in Fig. 1 for $\mathrm{Na}_{n}$ clusters. As expected from the theoretical considerations mentioned above the most abundant sizes are those with spherical shell closings at $n=8(1 \mathrm{~s}+1 \mathrm{p})$ or $n=20(1 \mathrm{~s}+1 \mathrm{p}+1 \mathrm{~d}+2 \mathrm{~s})$. However, such a conclusion can, in general, not be drawn from measurements of this type, since the ionisation process itself may change the neutral size distribution completely through

$\begin{array}{ll}\text { Contents } & \\ \begin{array}{l}\text { Properties of Size Selected } \\ \quad \text { Neutral Clusters }\end{array} & 41 \\ \text { Electro-chemical Induced Fusion } & 45 \\ \text { Hollow Cathode Arcs } & 46 \\ \begin{array}{l}\text { European Astrophysics Doctoral } \\ \quad \text { Network }\end{array} & 51 \\ \begin{array}{l}\text { Physical Mechanisms in Polymer } \\ \text { Failure }\end{array} & 52 \\ \begin{array}{l}\text { ICFA Plots a New Course } \\ \text { Decisions of EPS Council }\end{array} & 54 \\ \text { Jugoslav Directory of Physicists } & 56 \\ \end{array}$



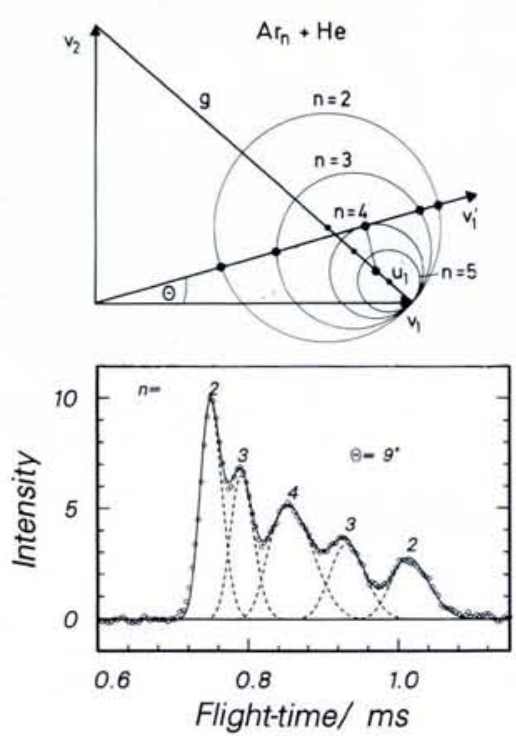

Fig. 2 - Schematic velocity vector diagram (not to scale) for the scattering of an $\mathrm{Ar}_{n}$ cluster beam $\left(v_{1}\right)$ from a He beam $\left(v_{2}\right)$. The circles give the velocities of elastically scattered dimers to pentamers around the different centres-of-mass. The lower part shows a measured time-of-flight spectrum taken at $\theta=9^{\circ}$ and the dimer mass $m=80$ amu along the laboratory velocity $v$, of the diagram. The maxima of the spectrum occurring at the crossing points with the circles represent the dispersed cluster species in the beam (from Ref. 3).

fragmentation. Experimental methods are required which label the different clusters according to their size independently of the ionisation process.

In this review we shall discuss such methods with emphasis on the separation by scattering from an atomic beam. Specific results will be presented on fragmentation, photo-dissociation of weakly bound clusters, and the chemical reactivity of metal clusters.

\section{Size Selection}

There are essentially three methods which have been used to date for size selecting neutral clusters in experiments. One is to apply spectroscopy. Here advantage is taken of the fact that different cluster sizes exhibit different spectral lines or shifts. A very recent development is the neutralisation of a cluster ion which can be selected easily in a mass spectrometer although care has to be taken to see that the charge exchange process does not generate too much excess energy which can lead again to fragmentation. In the scattering method, clusters are scattered from an atomic beam and separation takes place, since the lighter clusters are deflected into larger scattering angles with larger velocities than the heavier clusters $[3,4]$. This behaviour is demonstrated in the velocity vector diagram of
Fig. 2 where it is assumed that the cluster beam (velocity $v_{1}$ ) and the atomic beam $\left(v_{2}\right)$ intersect at an angle of $90^{\circ}$. The final centre-of-mass $(\mathrm{cm})$ velocities $u_{1}^{\prime}$ of the different cluster sizes are circles around the corresponding centre-of-masses with radii that decrease according to the increasing mass. The real distributions are obtained by taking a cut $v_{1}$ through these circles. Since the masses of the clusters are usually heavier than the mass of the scattering partner, there are in general two contributions with different velocities.

Detection is carried out in a mass spectrometer tuned to a particular mass to reduce background. There are two modes of operation for the cluster separation. The choice of the scattering angle exlcudes the larger clusters by kinematical constraints ( $n>4$ in Fig. 3). The smaller clusters $(n<4)$ are separated by mass selection. For this method to work, at least a small fraction of the cluster $\mathrm{Ar}_{4}$ must appear as ion $\mathrm{Ar}_{4}^{+}$. If, however, the $\mathrm{Ar}_{4}$ cluster fragments to $\mathrm{Ar}_{2}^{+}$, the different arrival time distinguishes those ions originating from $\mathrm{Ar}_{4}$, $\mathrm{Ar}_{3}$ or $\mathrm{Ar}_{2}$. The latter method is independent of the special detection scheme, since the angle and the velocity determine completely the cluster size. High angular and velocity resolution are required and these can now be obtained. As an example of the second procedure, the time-of-flight spectrum for $\mathrm{Ar}_{n}$ clusters scattered from $\mathrm{He}$ is shown in Fig. 2 as seen by a measurement set for dimers. At $\theta=9^{\circ}$ we observe contributions from dimers, trimers and tetramers as we should expect from the diagram. It is clear that extensive fragmentation has taken place.

\section{Ionisation}

The fragmentation consequent upon the ionisation of the cluster is easily measured by the scattering method. Once the deflection angle and the velocity after the collision are specified, the beam will contain only clusters of size $n$ and measurement of the product ions at masses $k$ gives the fragmentation probability $f_{n k}$. The systems investigated so far by this technique are the van der Waals clusters $\mathrm{Ar}_{n},\left(\mathrm{CO}_{2}\right)_{n},(\mathrm{NO})_{n}$ and $\left(\mathrm{C}_{2} \mathrm{H}_{4}\right)_{n}$ and the quasi hydrogen bonded $\left(\mathrm{NH}_{3}\right)_{n}$ ionised by electron bombardment [4]. For the first three systems, the fragment ion masses are simply multiples of the monomer mass. The results are given in Table 1. The fragmentation pattern is completely dominated by either the dimer $\left(\mathrm{Ar}_{2}^{+}\right)$or the monomer $\left(\mathrm{CO}_{2}^{+}, \mathrm{NO}^{+}\right)$product ions, in spite of the
Table 1 - Fragmentation by lonisation

\begin{tabular}{|lllll|}
\hline Cluster & $\boldsymbol{n}$ & Main lon $\boldsymbol{k}$ & $\boldsymbol{f}_{n k}$ & Ref. \\
$\mathrm{Ar}_{n}$ & $2-6$ & $\mathrm{Ar}_{2}^{+}$ & $0.6-1.0$ & 4 \\
$\left(\mathrm{CO}_{2}\right)_{n}$ & $2-3$ & $\mathrm{CO}_{2}^{+}$ & $0.8-0.9$ & 4 \\
$(\mathrm{NO})_{n}$ & $2-6$ & $\mathrm{NO}^{+}$ & $>0.9$ & 4 \\
$\left(\mathrm{C}_{2} \mathrm{H}_{4}\right)_{n}$ & $2-4$ & $\mathrm{C}_{4} \mathrm{H}_{8}^{+}, \mathrm{C}_{3} \mathrm{H}_{5}^{+} \cong 0.6$ & 4 \\
$\left(\mathrm{NH}_{3}\right)_{n}$ & $2-5$ & $\mathrm{NH}_{4}^{+}$ & $0.5-0.7$ & 4 \\
$\mathrm{Na}_{n}$ & $2-21$ & $\mathrm{Na}_{n}^{+} \mathrm{Na}_{n-1}^{+}$ & $0.7-0.9$ & 6 \\
\hline
\end{tabular}

fact that the investigated parent clusters included sizes up to $n=6$. The most remarkable feature for $\mathrm{Ar}_{n}$ clusters is the strong preference for the dimer ion. No $\mathrm{Ar}_{3}^{+}$ions are produced from parent clusters of the same mass and even clusters of $n=4,5$, and 6 fragment with a probability of $>0.95$ to $\mathrm{Ar}_{2}^{+}$. The first contribution to $\mathrm{Ar}_{3}^{+}$comes from pentamers.

The explanation behind all these findings lies in the change in geometry and energetics when going from neutral clusters in a direct transition to ionized clusters [5]. The neutral clusters are van der Waals systems with large minimum distances and binding energies between 12 and $70 \mathrm{meV}$, whereas the ionised clusters exhibit binding energies in the order of 0.6 to $1.2 \mathrm{eV}$ and the minimum distances are shifted to smaller values. For rare gas clusters, a general mechanism has been proposed. After ionisation, charge localisation occurs for the dimer (or slightly larger cluster ions), which carries, e.g. for $\mathrm{Ar}_{2}^{+}$, an excess energy of $1.2 \mathrm{eV}$. This energy is released to the cluster by vibrational coupling leading to the evaporation of atoms and thus heavy fragmentation. Molecular dynamics calculations confirm this picture showing rapid energy relaxation on a time scale of several hundred picoseconds and the evaporation of several atoms.

The molecular systems $\left(\mathrm{C}_{2} \mathrm{H}_{4}\right)_{n}$ and $\left(\mathrm{NH}_{3}\right)_{0}$ behave differently. $A$ large number of fragment channels appear which are caused by internal ion-molecule reactions between an initially ionised monomer molecule and its molecular partners within the clusters. For ethylene, the highly excited $\mathrm{C}_{4} \mathrm{H}_{8}{ }^{+}$ion plays the main role in determining the fragmentation pattern. For ammonia it is the protonated $\mathrm{NH}_{4}^{+}$ion whose excess energy is used to evaporate further $\mathrm{NH}_{3}$ units in addition to the loss of the $\mathrm{NH}_{2}$ fragment. What is common to all these systems is the intrinsic nature of fragmentation because of the large geometrical change upon ionisation. It cannot be avoided either by choosing a different ionisation scheme or by adjusting the threshold energy. 
However, these factors should not play a role in systems where a geometrical change does not occur, since the electrons are largely delocalised, as is the case for metal clusters. Very recent experiments for $\mathrm{Na}_{n}$ clusters which are produced by charge exchange from $\mathrm{Na}_{n}^{+}$ + Cs show indeed this behaviour [6]. By carefully choosing the photon energy for ionisation, the fragmentation is found to be very small with the exception of $\mathrm{Na}_{9}$ and $\mathrm{Na}_{21}$ for which the evaporation of one atom is mainly found. This result is attributed to the internal excitation of the size selected neutrals produced by the charge transfer and the relative stability of the products $\mathrm{Na}_{8}$ and $\mathrm{Na}_{20}$ because of the closed electron shells.

\section{Infrared Photodissociation}

The infrared photo-dissociation of weakly bound clusters has attracted much interest in recent years and a variety of systems have been studied. In these experiments, a vibrational mode of the molecular component is excited by infrared laser photons. If the photon energy is larger than the binding energy of the complex, the complex predissociates. Typically, the clusters are prepared in a supersonic expansion and the dissociation is measured by monitoring the depletion of the molecular beam as a function of the laser frequency. The line shift of these dissociation spectra is caused by the interaction of the molecular oscillator with the surrounding cluster molecules and this gives information on the structure of the cluster. In contrast, the line width, if homogeneously broadened, gives information on the lifetime and thus on the dynamical coupling of the vibrational molecular mode to the internal modes of the cluster motions. The use of size selected clusters in these experiments allows us for the first time to extend the experiments to species other than dimers and trimers.

The experimental arrangement and results for $\left(\mathrm{CH}_{3} \mathrm{OH}\right)_{n}$ clusters are shown in Fig. 3. First the cluster beam is scattered from $\mathrm{He}$. By measuring the intensity at different angles and masses, different cluster sizes are selected. Then the methanol cluster is dissociated by the infrared radiation of a line tunable $\mathrm{CO}_{2}$-laser, collinear to the scattered cluster beam. The dissociation after the excitation of the $\mathrm{C}-\mathrm{O}$ stretch mode near $1034 \mathrm{~cm}^{-1}$ is observed from the dimer to the octamer. The spectra of these clusters of the hydrogen bonded system are different. Three of them are displayed in Fig. 3 [7]. The dimer shows a two peak structure with one peak shifted to the

\section{University of Geneva}

announces an opening for a position of a

FULL PROFESSOR in

\section{COMPUTATIONAL CONDENSED MATTER PHYSICS}

The new professor will have the responsibility for research and teaching in computational condensed matter physics. He will in particular have the task to develop the research activities of the "Institut Romand de Recherches Numériques en Physique des Matériaux" (IRRMA) situated at the campus of the Ecole Polytechnique Fédérale de Lausanne. He will assume the direction of this Institute for a certain number of years and it is also expected that he actively participates in the scientific and academic life of the University of Geneva.

The applicant should have a Ph.D. in physics or an equivalent degree. Experience in teaching, leading and management of research groups as well as some experience in university administration is desirable.

Letters of application, a curriculum vitae and a list of publications should be addressed before 30 June 1989 to:

Secrétariat de la Faculté des Sciences,

20, quai Ernest-Ansermet, CH-1211 Geneva 4, Switzerland, where additional information may be obtained. red and one to the blue compared with the gas phase monomer frequency. This behaviour is traced back to the two nonequivalent positions of $\mathrm{CH}_{3} \mathrm{OH}$ in the dimer, which also leads to a large dipole moment. The clusters from $n=3$ to $n=5$ show single peaks which are continuously shifted to the blue compared with the monomer frequency. This is a clear indication of equivalent $\mathrm{CH}_{3} \mathrm{OH}$ units which form planar rings in agreement with theoretical predictions and their vanishing dipole moment. The hexamer again shows a two peak structure which is also observed for the larger clusters. One peak continues to be

\section{Physica
Scripta}

An international journal for

experimental and theoretical physics

Vol. T26 1989

\section{Proceedings of the 20th EGAS Conference}

European Group for Atomic Spectroscopy - A section of the EPS Division for Atomic and Molecular Physics

\section{Graz, Austria, 12-15 July 1988 Edited by L. Windholz}

Eleven of the 13 invited lectures prepared for the meeting which attracted 250 spectroscopists from 25 countries.

Following a history of EGAS by P.F.A. Klinkenberg are the contributions of W. Hogenvorst et al., S.G. Dinev, Z.B. Rudzikas and J.V. Ciplys, B.R. Judd, G. von Oppen, B. Denne, P.M. Koch et al., U.I. Safronova, H. Jäger, M. Broyer, S. Svanberg.

100 pages in all

Orders to Physica Scripta at the address shown below :

Price: 250.- SKR or 40.- US\$.

Published by the

ROYAL SWEDISH ACADEMY OF SCIENCES

Box 50005

ISSN 0281-1847

S- 10405 Stockholm / Sweden 

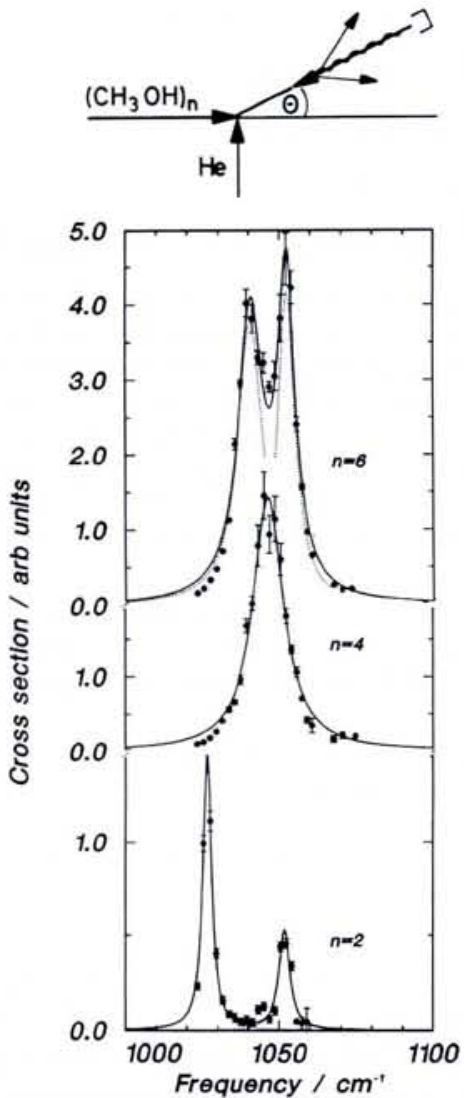

Fig. 3 - Experimental arrangement and measured frequency dependences of the infrared photodissociation cross-section of methanol clusters, size selected by the scattering method (from Ref. 7).

shifted to the blue as is observed for the smaller clusters and the other is shifted to the red by about $10 \mathrm{~cm}^{-1}$. From the spectrum of the hexamer a structural change can be inferred.

Theoretical calculations based on multi-dimensional total energy surfaces indicate that, in addition to the most stable form (the planar rings), isomers are found with strong deformations of the ring. Therefore we assign these two peaks which first appear for the hexamer to two different isomers, one of which is close to a planar ring and the other corresponding to a distorted ring structure.

Similar experiments carried out for the van der Waals clusters $\left(\mathrm{C}_{2} \mathrm{H}_{4}\right)_{n}$ show nearly the same dissociation spectra for the dimer up to the hexamer after the excitation of the out-of-plane $v_{7}$ mode [8]. We attribute these findings to a chainlike structure of the ethylene clusters which implies that in this case, only the nearest neighbours are affected by the dissociation leading to very similar results for all clusters investigated. These studies clearly show that infrared photodissociation spectra of size selected molecular clusters contain detailed information about the structure of the clusters.

\section{Chemical Reactivity}

Among the properties of small metal clusters that have recently become accessible to experimental studies are their chemical interactions with simple gas phase molecules. Clusters of transition metal atoms offer the prospect of studying chemical reactions on surfaces with obvious applications in heterogeneous catalysis. A typical example is the study of the reaction of ion clusters $\mathrm{Fe}_{n}$ with hydrogen molecules $\mathrm{H}_{2}$. The clusters are generated by pulsed laser evaporation from a metal rod, enter a flowtube reactor where $\mathrm{H}_{2}$ is added, and are detected in a laser-ionisation time-offlight mass spectrometer [9]. In the simplest experiments the disappearance of the bare cluster signal in the mass spectrum is monitored as the quantity of the reactant gas is increased. In this way, rate constants for the addition of the first $\mathrm{H}_{2}$ are extracted which are shown in Fig. 4.

The variation of the reactivity with cluster size is highly non-monotonic and varies by over three orders of magnitude between $\mathrm{Fe}_{8}$ and $\mathrm{Fe}_{23}$. Large changes occur between $\mathrm{Fe}_{8}$ and $\mathrm{Fe}_{9}, \mathrm{Fe}_{14}$ and $\mathrm{Fe}_{16}, \mathrm{Fe}_{18}$ and $\mathrm{Fe}_{19}$. The final jump in the rate constant between $\mathrm{Fe}_{22}$ and $\mathrm{Fe}_{23}$ is to a value close to that predicted by the bulk iron sticking probability, so that in this case, bulk behaviour is reached for $n=23$.

The cause of the strong reactivity dependence on cluster size has been a subject of considerable discussion. Measurements of the ionisation potentials of iron clusters, also presented in Fig. 4, show a dependence on cluster size that nearly anti-correlates with the reactivity: high ionisation potentials give low rates and vice versa [10]. This correlation supports a model for which the reaction procedes via dissociated chemisorption of $\mathrm{H}_{2}$. This interaction involves electron donation from the metal cluster into antibonding orbitals of $\mathrm{H}_{2}$, thus weakening the $\mathrm{H}_{2}$ bond. This process becomes more probable the lower the ionisation potential of the cluster is.
However, experiments of other groups and on other systems indicate that this simple correlation is somewhat less compelling and not the entire picture. It is believed that a significant structural change in cluster geometry is finally responsible for the drastic changes in reactivity.

The selected examples demonstrate that substantial progress has been made in the efforts to experiment with size selected clusters. On the other hand, direct experimental information on cluster structure and cluster temperatures over a wide range of sizes is still difficult to achieve and methods are just in the state of development. It is hoped that we shall get more complete answers in the future by a combined effort from molecular and solid state physics, chemistry and materials science which are all involved in cluster research.

\section{REFERENCES}

[1] Journals and books for further reading: Ber. Bunsenges. Phys. Chem. 88 (1984) 188-322;

Surf. Sci. 156 (1985) 1-562

Z. Phys. D 3 (1986) 95-337;

J. Phys. Chem. 91 (1987) 2447-2671;

Elemental and Molecular Clusters, Eds. G. Benedek, T.P. Martin and G. Pacchioni (Springer, Berlin) 1987.

[2] Knight W.D., de Heer W.A. and Saunders W.A., Z. Phys. D 3 (1986) 109.

[3] Buck U. and Meyer H., J. Chem. Phys. 84 (1986) 4854

[4] Buck U., J. Phys. Chem. 92 (1988) 1023.

[5] Haberland H., Surf. Sci. 156 (1985) 305.

[6] Brechigniac C., Cahuzac Ph., Leygnier J., Pflaum R. and Weiner J., Phys. Rev. Lett. 61 (1988) 314.

[7] Huisken F. and Stemmler M., Chem. Phys. Lett. 144 (1988) 391; Buck U., GuX.J., Lauenstein $\mathrm{Ch}$. and Rudolph A., J. Phys. Chem. 92 (1988) 5561.

[8] Buck U., Huisken F., Lauenstein Ch., Meyer H. and Sroka R., J. Chem. Phys. 87 (1987) 6276

[9] Parks E.K., Weiller B.H., Bechthold P.S., Hoffmann W.F., Nieman G.C., Pobo L.G. and Riley S.J., J. Chem. Phys. 88 (1988) 1622. [10] Whetton R.L., Cox D.M., Trevor D.J. and Kaldor A., Phys. Rev. Lett. 54 (1985) 1494.
Fig. 4 - Measured rate constants for the reaction of iron clusters with hydrogen (left scale, from Ref. 9) and iron cluster ionisation potentials (right scale, from Ref. 10).

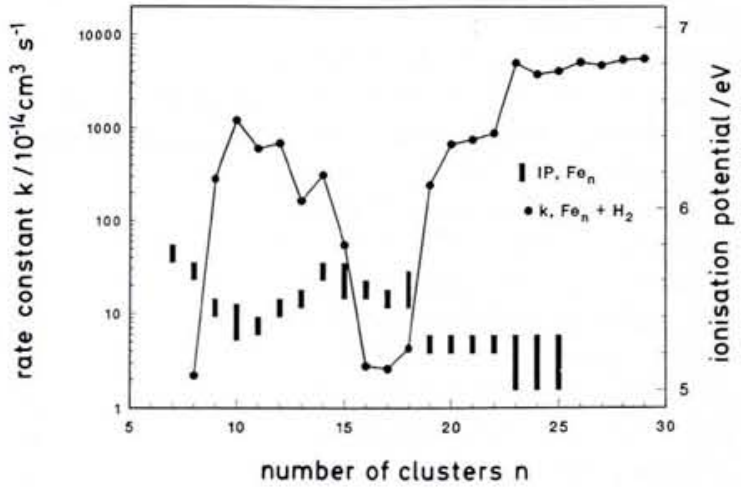

